\title{
Light yield measurements in a liquid scintillator detector with wavelength-shifting fibre readout
}

\author{
M. Doucet ${ }^{a}$, J.-P. Fabre ${ }^{a}$, G. Grégoire ${ }^{b}$ J. Panman ${ }^{a}$, P. Zucchelli ${ }^{a, c}$ \\ ${ }^{a}$ CERN, Geneva, Switzerland \\ ${ }^{b}$ Université Catholique de Louvain, Louvain-la-Neuve, Belgium \\ ${ }^{c}$ On leave of absence from INFN - Sezione di Ferrara
}

\begin{abstract}
A technique based on liquid scintillator with wavelength-shifting fibre readout is interesting for large-mass neutrino detectors. In this paper, we present results obtained with a laboratory prototype assembled at CERN. Mixtures made of economical chemical components were compared to commercial liquid scintillators. The light yield was also studied as a function of fibre diameter, fibre position, and surface quality of the container.
\end{abstract}

(To be submitted to Nucl. Instr. and Meth.) 


\section{Introduction}

A technique based on liquid scintillator with wavelength-shifting fibre readout has been identified as a promising solution for large-mass neutrino experiments [1]. A single container of liquid scintillator segmented in tubes by optical separators can provide a granularity of a few centimetres over a large volume. This volume can be read out by wavelength-shifting (WLS) fibres, hence reducing the surface to be equipped with photo-detectors. If one can in addition make the tubes very long, the number of photosensitive devices necessary to read the volume of the detector can be reduced as the detector is made wider. The maximal length of the tubes is dominated by the attenuation length of the WLS fibres and the minimal number of photo-electrons that need to be detected to perform the physics measurements. First measurements using this type of detector were reported in a previous publication [1]. In this paper, we describe light yield measurements obtained using different types of liquids, fibres and reflecting surfaces of the liquid scintillator tube.

The first aim of the present study is to identify good, inexpensive and safe liquid scintillators that could be used in large quantities. For this purpose, we have chosen to test two commercial chemicals as base liquid. One is Linear Alkylbenzene (LAB) [2], and the second is naphthenic oil [3] (Nyflex200). These two liquids have high flash points $\left(154^{\circ} \mathrm{C}\right.$ and $141^{\circ} \mathrm{C}$ respectively) and are economical commercial products that do not degrade the fibre quality. In addition, they do not represent any health or environmental hazard. As secondary liquids, we compared Phenyl-o-xylylethane (PXE) [4] and pseudocumene (PC). PXE is also a safe commercial product. It has a flash point of about $145^{\circ} \mathrm{C}$. For the present purpose, it constitutes a good alternative to pseudocumene, which has a flash point of only $43^{\circ} \mathrm{C}$. In a pure state, both of these secondary liquids, although good scintillators, will degrade WLS fibres after prolonged immersion. On the other hand, the lifetime of WLS fibres have been measured to be longer than several years when the amount of those secondary liquids is kept sufficiently low. For example, the lifetime of fibres in Bicron BC-517L liquid containing about 30\% pseudocumene has been estimated to be over 15 years [5]. As solutes to be mixed with the liquids, we used PMP and PPO. Several mixtures were tested and the light yield measured. Our own mixtures were compared to two commercial liquid scintillators: Bicron BC-517L [6] and EJ-399-04 from Eljen Technology [7]. The liquid from Eljen Technology, which we will denote EJ, is made of $90 \% \mathrm{LAB}$ and $10 \%$ PXE in addition to solutes unknown to us. BC-517L and EJ have flash points of $102{ }^{\circ} \mathrm{C}$ and $138{ }^{\circ} \mathrm{C}$ respectively. In addition, a sample of liquid scintillator used in the MACRO experiment [8] was tested. The compositions of the mixtures that were mixed at CERN and of the MACRO scintillator are reported in table 1.

The second aim of this work is to study the light yield as a function of three parameters: the fibre position within the liquid scintillator volume, the fibre diameter, and the reflecting surface of the tube containing the scintillator. For this purpose, WLS fibres of $1.2 \mathrm{~mm}$ and $0.5 \mathrm{~mm}$ diameter were placed in the liquid at different positions. Three tubes were used to study qualitatively the effect of the reflecting surface. They 
were tubes having either a bare aluminium inner surface, an aluminium inner surface painted with reflector paint, or a white PVC surface.

\section{$2 \quad$ Experimental setup}

Figure 1 shows a sketch of the experimental setup. The apparatus has already been described in a previous publication [1]. Three tubes were used for the measurements, each having a length of 1 metre:

1. An aluminium tube having an outer cross-section of $3 \times 3 \mathrm{~cm}^{2}$ and painted on the inside surface with Bicron $\mathrm{BC}-622 \mathrm{~A} \mathrm{TiO}_{2}$ reflector paint. The aluminium had a thickness of $3 \mathrm{~mm}$, so that the cross-section of the volume of the liquid filling the tube was $2.4 \times 2.4 \mathrm{~cm}^{2}$.

2. An aluminium tube identical in dimensions to the first one, with an inner surface cleaned by chemical passivation in order to provide a uniform reflective surface.

3. A commercial tube of extruded white PVC [9]. The tube containing the liquid scintillator was part of a larger structure of tubes of which only one was used. The inner cross-section of the tube was $5.2 \times 2.9 \mathrm{~cm}^{2}$.

The tubes were placed consecutively in a common setup. They were held on each end by a light-tight end-cap anodized on the inside with a black coating. Each endcap held a photomultiplier tube (PMT) in a vertical position. The PMTs used were Hamamatsu R1635P with bialkali photocathode. The quantum efficiency of this PMT is about $10 \%$ at $550 \mathrm{~nm}$. The ends of the tube in the setup were closed by an aluminium wall painted with reflector paint. This aluminium piece had a set of holes in order to change the fibre position. The fibres could run through the centre of the tube, either in the middle of the liquid volume (we will denote this position ctr) or lying on the lower surface (bot-ctr), or they could run through a corner on the bottom surface of the tube (bot-cor). Kuraray Y11-MSJ200 [10] fibres were placed inside the tube. Fibres having diameters of $0.5 \mathrm{~mm}$ and $1.2 \mathrm{~mm}$ were used. The polished ends of the fibre were applied to the window of a PMT by a teflon cap. To reach the PMTs, the ends of the fibre extended about $30 \mathrm{~cm}$ from the tube on each side and were bent with a radius of curvature of about $10 \mathrm{~cm}$. For a given liquid, the apparatus was filled such that the tube was full.

Two EJ liquids were used. One was used for the study of the effect of fibre positions, fibre diameter and reflective surfaces. It had been in the setup for several months during the test period before data taking, and its light yield was later found to be low compared to the same liquid when taken fresh from its delivery bottle. Throughout this paper, we will label the degraded EJ-399-04 EJ (old) and the fresh EJ-399-04 EJ (new). 


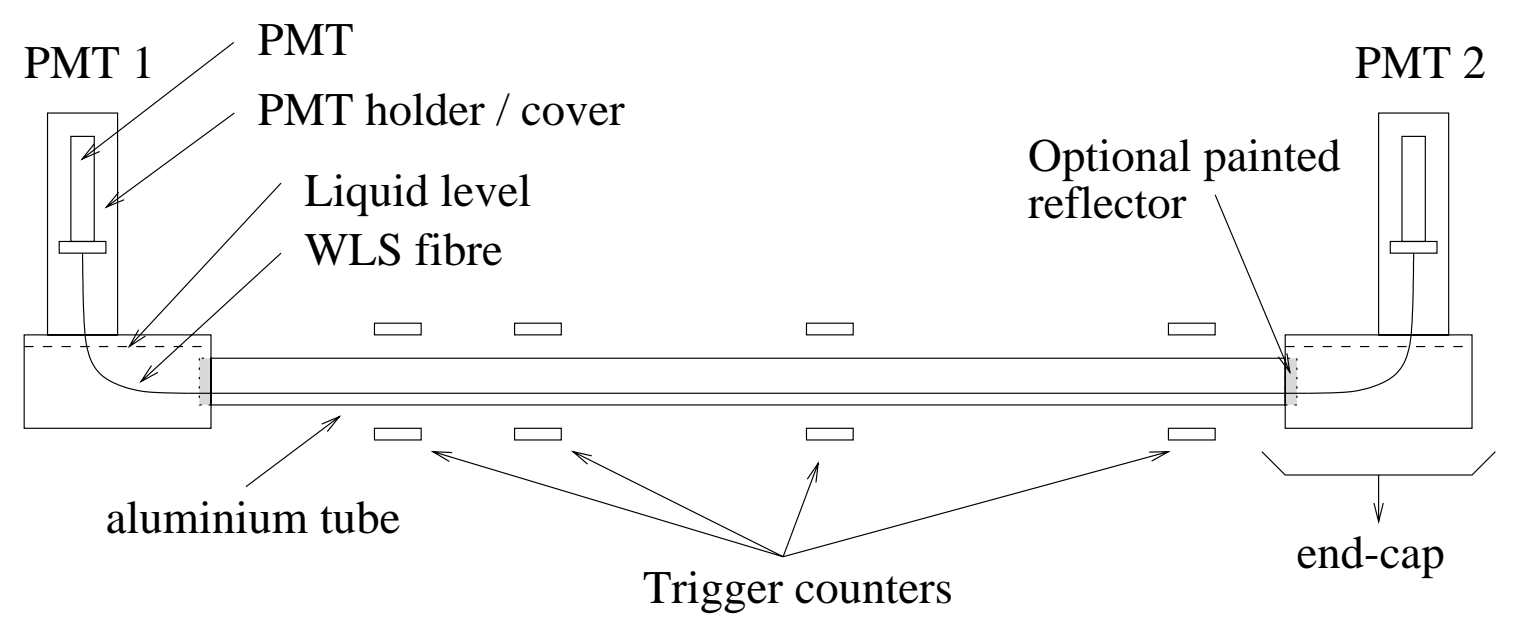

Figure 1: Schematic view of the experimental setup.

For the study of liquids, the EJ liquids (both old and new) were compared to Bicron BC-517L and to the scintillators listed in table 1. Before testing a new liquid, the apparatus was cleaned with alcohol. To ensure that the conditions of all the liquids were the same, the liquid scintillators that were mixed at CERN were placed under vacuum for three days before being tested in order to remove oxygen. Apart from the EJ (old) liquid, all liquids that were not mixed at CERN were tested using samples taken directly from their delivery bottle. We have not quantified the effect of the degradation of the liquids when exposed to air.

The tests were performed with cosmic-ray muons. For the trigger, four identical pairs of scintillator pads read by 1.5 inch PMTs were placed along the tube. For each pair, the pads were placed above and below the tube to provide a cosmic-ray trigger. The distances between the pads and the centre of the tube were $6.5 \mathrm{~cm}$ (above) and $5.5 \mathrm{~cm}$ (below). The scintillator pads of the trigger system had a surface of about $5 \mathrm{~cm} \times 2 \mathrm{~cm}$ and a thickness of $5 \mathrm{~mm}$. For each cosmic trigger, the signal of each of the ten PMTs (two for the fibre and $2 \times 4$ for the trigger counters) was read. The trigger pairs were placed along the tube to scan its length. Only the trigger pair placed at the central position of the tube (50 cm from each PMT) provided the measurements for the results. The other pairs, placed at $92 \mathrm{~cm}, 62 \mathrm{~cm}$ and $22 \mathrm{~cm}$ from PMT 1 , were only used to evaluate systematic errors. All PMT signals were read by means of Lecroy 1182 VME ADCs. The VME crate was connected to a desktop PC by optical fibres through a Bit3-618 PCI-VME interface [11]. The trigger rate of the setup was 2-3 counts per minute per scintillator pad pair. 


\section{Methodology}

The light yield was measured on both sides of the fibre by the photon-counting PMTs. Examples of signal distributions are shown in figures $2 \mathrm{a}$ and b. Pedestal subtraction has been applied to these distributions. The pedestal for each PMT was measured in an independent run. It was found to be stable within its half-width throughout the data taking period. The data shown in figure 2 were taken using the EJ (new) liquid, with a $1.2 \mathrm{~mm}$ fibre passing through the centre of the painted tube. The average light yield on each side was measured by fitting a Gaussian to the distribution of the logarithm of the ADC counts, which approximates the shape of a Landau distribution (figures $2 \mathrm{c}$ and d). To evaluate the average number of photo-electrons on each PMT corresponding to a given data run, the distribution of the ratio of the pedestal subtracted signals on both sides for all events was used (figure 2e). For this purpose, the signal on each side was scaled by its average light yield for the run so that the ratio was centred at unity, to take into account possible gain variations. The effect of a larger average number of photo-electrons is to narrow the ratio distribution. In the approximation of a large number of photo-electrons, for particles passing through the middle point of the tube, the width of the ratio distribution is related to the number of photo-electrons on each PMT by

$$
\frac{\sigma(R)}{<R>}=\sqrt{\frac{2}{N_{\mathrm{pe}}}},
$$

where $\sigma(R)$ is the width of the distribution assuming a Gaussian fit, $\langle R\rangle$ is the average value of the distribution (in the present case put to unity), and $N_{\text {pe }}$ is the average number of photo-electrons on each PMT, which is assumed to be identical for both PMTs. Since some of our configurations provide a very low light yield, this approximation was not used but a simple Monte Carlo simulation of the statistical effects was used instead. The program simulated the distribution of the ratio of photoelectrons collected on the two PMTs according to fluctuations in the initial light emitted by the scintillator and in the photo-electrons emitted by the photo-cathode. The simulated distributions obtained for various average numbers of photo-electrons were compared to the measured ratio distribution using a Kolmogorov-Smirnov test. The confidence level distribution given by the Kolmogorov-Smirnov test as a function of the average number of photo-electrons was then fitted to a Gaussian and the average value of the fit was taken as the average number of photo-electrons of the data (figure $2 \mathrm{f}$ ).

Systematic errors were evaluated to estimate the reproducibility of the measurements. The systematic errors from the light yield were evaluated from the geometric average $^{1}$ of the mean signals measured on the two PMTs. The three additional trigger pairs were used for this purpose, and the geometric average calculated at these positions were compared to the one measured at the central position. A conservative $10 \%$ was taken as systematic error.

The effect of the position of the pedestal was also evaluated by varying the pedestal

\footnotetext{
${ }^{1}$ The geometric average of the signals of the two PMTs is defined as $\sqrt{\text { signal } 1 \times \text { signal } 2}$.
} 
value by twice its Gaussian standard deviation and computing the variation in geometric average. The effect was found to be of the order of $2 \%$ for low light yield configurations and negligible for high light yield configurations.

\section{Light yield as a function of liquids}

A set of measurements was performed using ten different liquids. Two of these were the EJ liquids (labelled old and new), one was the BC-517L, and the remaining seven are listed in table 1. Each liquid was tested in the same conditions. The aluminium tube painted with the Bicron reflector paint was used and a $1.2 \mathrm{~mm}$ diameter fibre was placed in the centre of the liquid volume.

For each liquid, data runs of about three days were taken and the signal on each PMT was measured for each cosmic trigger. The average number of photo-electrons on each PMT was measured using the technique described above. Figure 3 shows the results obtained. As a check of the calibration, figure 3a shows the ADC count on each PMT as a function of the fitted number of photo-electrons for each run. A linear fit of these data gives calibrations for this sample of runs of $14.0 \pm 0.4$ and $12.0 \pm 0.4$ ADC counts per photo-electron for PMT 1 and PMT 2 respectively. These calibration values agree with the ones calculated from the documentation provided by Hamamatsu, which are about 13.0 ADC counts per photo-electron for each PMT. The signal on the two PMTs for each liquid is shown in figure $3 \mathrm{~b}$. Figure $3 \mathrm{c}$ shows the measured average number of photo-electrons on the PMTs for the same conditions. The data points are reported in table 2 .

The results obtained show that it is possible to produce good liquid scintillators from economical industrial chemicals. Although the EJ (new) liquid, which is based on the components we have studied, has a higher light yield than all our mixtures, some of our mixtures have a light yield comparable to BC-517L. We note the following observations:

- As primary liquids, both naphthenic oil (Nyflex 200) and LAB provide a good base material. It should be mentioned that not all naphthenic oils are equally adequate for this purpose. We present here the oil with the best scintillating properties among the Nynas naphthenic oils that were made available to us ${ }^{2}$.

- As secondary liquids, the comparison between oil+PC+PMP and oil+PXE+PMP indicates that PXE can provide a good replacement for the commonly used PC, at least when used in conjunction with naphthenic oil. Used with LAB, we find that PXE does not produce any sizable gain in light yield compared to LAB used without secondary liquid. This is not the case with naphthenic oil, for which the

\footnotetext{
${ }^{2}$ The emission spectra of different oils in which PMP had been dissolved were compared to determine the liquid to be used. Nyflex209, 222B, 809, 810 and Nytex809, 810 and 820 were compared.
} 
addition of a secondary liquid like PC or PXE causes a gain of about a factor two in light yield. This would indicate that the emission and absorption spectra of LAB and PXE are not matched to produce a large increase in the overall scintillation efficiency of the LAB+PXE+PMP mixture.

- The solute should be matched with the absorption spectrum of the WLS fibre, which peaks between 400 and $450 \mathrm{~nm}$. The comparison between LAB+PXE+PMP and $\mathrm{LAB}+\mathrm{PXE}+\mathrm{PPO}$ demonstrates this point. PPO emits mostly below $400 \mathrm{~nm}$. The MACRO scintillator has bis-MSB as a secondary solute in addition to PPO. A measurement of the scintillation spectrum of this scintillator showed that it emits mostly below $400 \mathrm{~nm}$. It should be noted that this scintillator was optimised for a different type of detector, for which the scintillation light was not transported through a WLS fibre but was measured directly.

\section{$5 \quad$ Light yield as a function of fibres and surfaces}

A set of measurements was performed using the EJ (old) liquid with the two aluminium tubes, in all combinations of fibre positions and fibre diameters. For each configuration, data runs of about three days were taken and the signal on each PMT was measured for each cosmic trigger. The average number of photo-electrons on each PMT was measured using the technique described above. Figure 4 shows the results obtained. As a check of the calibration, figure 4a shows the ADC count on each PMT as a function of the fitted number of photo-electrons. A linear fit of these data gives calibrations for this sample of runs of $13.1 \pm 0.4$ and $12.5 \pm 0.4$ ADC counts per photo-electron for PMT 1 and PMT 2 respectively.

The signal on each PMT for each running condition is shown in figure $4 \mathrm{~b}$. These samples are separated in groups of three points; one group for each reflective surface and fibre diameter. The first is for the fibre lying on the bottom surface of the tube, running through the centre. The second is for the fibre running through a corner of the bottom surface. The third is for the fibre running through the centre of the liquid volume. Four conditions of fibre diameter and reflective surface are presented: aluminium reflector with $1.2 \mathrm{~mm}$ or $0.5 \mathrm{~mm}$ diameter fibres, and aluminium painted with the Bicron reflector paint with $1.2 \mathrm{~mm}$ or $0.5 \mathrm{~mm}$ diameter fibres. Figure $4 \mathrm{c}$ shows the measured average number of photo-electrons for the same conditions. The data points are reported in table 3.

A set of measurements was performed using the EJ (new) liquid with the PVC tube, in all combinations of fibre positions and fibre diameters. Figure 5 shows the results obtained. As a check of the calibration, figure $5 \mathrm{a}$ shows the ADC count on each $\mathrm{PMT}$ as a function of the fitted number of photo-electrons. A linear fit of these data gives calibrations for this sample of runs of $13.2 \pm 0.6$ and $11.7 \pm 0.5 \mathrm{ADC}$ counts per photo-electron for PMT 1 and PMT 2 respectively. 
The signal on each PMT for each running condition is shown in figure 5b. These samples are separated in groups of three points; one group for each reflective surface and fibre diameter. The first is for the fibre lying on the bottom surface of the tube, running through the centre. The second is for the fibre running through a corner of the bottom surface. The third is for the fibre running through the centre of the liquid volume. Figure $5 \mathrm{c}$ shows the measured average number of photo-electrons for the same conditions. The data points are reported in table 4 .

Although some fluctuations are observed, the ensemble of measurements does not show evidence that the light output is influenced by the position of the fibre within the tube, regardless of the reflecting surface and the fibre size. If there are any effects due to the fibre position, they should be of a relative size of at most $10 \%$, which is the relative error on the measurements.

For all types of reflecting surface, the light output is measured to be proportional to a factor at least as large as the ratio of the diameters of the fibres. Averaging over the three fibre positions, the ratio of geometric averages and the ratio of numbers of photo-electrons measured using $1.2 \mathrm{~mm}$ and $0.5 \mathrm{~mm}$ fibres are comparable to the ratio of fibre diameters in the case of the aluminium reflector and the reflective paint, and larger in the case of the PVC tube. These values are reported in table 5. Such effects could be caused by poor reflective properties of the PVC in the region of wavelengths corresponding to the absorption region of the fibre. For perfect light transmission in the liquid and perfect reflectivity of the surface, we would not expect any difference in light yield for different fibre sizes. For the case where most of the photons are absorbed on the surface of the tube, we expect a ratio of light yield between the value of the ratio of the fibre diameters (if the fibre absorbs the light with high efficiency, which means surface absorption) and the square of the ratio of the fibre diameters (if the fibre absorbs the light with low efficiency, which means volume absorption). Our measurements are between those extremes. The reflectivity of the Bicron paint is expected to be approximately independent of the wavelength above $450 \mathrm{~nm}$. The reflectivity of the aluminium surface is expected to be constant over the entire range of wavelengths we are considering.

The data taking in different conditions of reflecting surfaces shows that the effect of the quality of the reflective surface can be large. We observe a reduction of the light yield of a factor 2.5 between the painted tube and the bare aluminium. This reduction factor appears to be independent of the fibre diameter. Taking into account the relative light yield of the EJ (new) with respect to the EJ (old) scintillators, the light yield measured with the PVC tube is between 2 to 4 times less than with the painted tube depending on the fibre diameter.

In summary, the light yield has been shown to be independent of the fibre position within $10 \%$. The effect on the light yield of the fibre size and of the quality of the reflective surface are difficult to quantify since they depend on many parameters which we do not measure, like the absorption in the fibre, the absorption and the reflectivity 
of the tube surface. Nonetheless, the measurements would indicate that the influence of the fibre and of the reflective surface can be large and do not factorize.

\section{Conclusions}

We have measured the light yield of a liquid scintillator detector with wavelengthshifting fibre readout in different configurations. In a liquid volume of $2.4 \times 2.4 \times 100 \mathrm{~cm}^{3}$, we measured light yields of up to about 50 photo-electrons. We have investigated the possibility to use common commercial chemicals to produce economical and safe liquid scintillators. Among the chemicals tested, we found that LAB or naphthenic oil mixed with PXE provide good scintillating material when used with PMP. These scintillators are safe from the point of view of flammability and environmental hazard, and do not attack the fibre material. The light output obtained with these liquids is comparable to the commonly used BC-517L. Further adjustments of relative quantities of the components of these mixtures could be imagined.

We have studied the effect on the light yield of the position of the WLS fibre within the liquid scintillator volume. We found no evidence for a dependence of the light yield

on the fibre position. This conclusion is independent of the fibre diameter and the reflective surface of the tube.

We have investigated the influence of the fibre diameter on the light yield. We measured that the gain in light when increasing the fibre diameter is at least as large as the ratio of fibre diameters, but also depends on the reflective surface of the tube. The effect on the light yield of the reflective surface was studied qualitatively by using three different surfaces. $\mathrm{A} \mathrm{TiO}_{2}$ surface was measured to give at least 2.5 times more light than bare aluminium and white PVC.

\section{Acknowledgements}

We would like to thank Daniel Bourillot, Jean Dupraz, Georges Roiron and Rudolf Buhler for their indispensable help with the test setup. We are grateful to Bruno Jacquet of Nynas for discussing with us and providing us with oil samples. We would like to thank Dan Levin and Erik Katsavounidis from the MACRO Collaboration for providing us with a sample of their liquid scintillator. We would also like to thank Ioannis Papadopoulos for help with running the system. 


\section{References}

[1] M. Doucet et al., CERN-EP-2000-011, accepted by Nucl. Inst. and Meth. A, (2000).

[2] Huntsman Petrochemical Corporation, Houston, TX, USA. Linear Alkylbenzene, ALK229.

[3] Nynas S.A., Pantin, France.

[4] Koch Chemical Company, Corpus Christi, TX, USA.

[5] K.G. Young et al., Radiat. Phys. Chem. Vol. 41 (1993) 215.

[6] Bicron, Newbury, Ohio, USA.

[7] Eljen Technology, Sweetwater, TX, USA.

[8] MACRO Collaboration, S. Ahlen et al., Nucl. Inst. and Meth. A 324 (1993) 337.

[9] Maine Plastiques, Ambrières les Vallées, France.

[10] Kuraray Co., Ltd., Tokyo, Japan.

[11] SBS Technologies, Inc., Albuquerque, NM, USA. 
Table 1: Composition of the MACRO liquid scintillator and of the liquid scintillators mixed at CERN. The amounts of liquids are given as volume fractions.

\begin{tabular}{|c|c|c|c|}
\hline Liquid & Primary liquid & Secondary liquid & Solute \\
\hline MACRO & 96.4\% paraffin oil & $3.6 \% \mathrm{PC}$ & $\begin{array}{l}1.44 \mathrm{~g} / \mathrm{l} \mathrm{PPO} \\
+1.4 \mathrm{mg} / \mathrm{l} \text { bis-MSB }\end{array}$ \\
\hline oil+PC+PMP & $75 \%$ Nyflex200 & $25 \% \mathrm{PC}$ & $2 \mathrm{~g} / \mathrm{l} \mathrm{PMP}$ \\
\hline oil+PXE+PMP & $75 \%$ Nyflex200 & $25 \% \mathrm{PXE}$ & $2 \mathrm{~g} / \mathrm{l} \mathrm{PMP}$ \\
\hline $\mathrm{LAB}+\mathrm{PXE}+\mathrm{PMP}$ & $75 \% \mathrm{LAB}$ & $25 \% \mathrm{PXE}$ & $2 \mathrm{~g} / \mathrm{l} \mathrm{PMP}$ \\
\hline $\mathrm{LAB}+\mathrm{PXE}+\mathrm{PPO}$ & $75 \% \mathrm{LAB}$ & $25 \% \mathrm{PXE}$ & $2 \mathrm{~g} / \mathrm{l} \mathrm{PPO}$ \\
\hline $\mathrm{LAB}+\mathrm{PMP}$ & $100 \% \mathrm{LAB}$ & - & $2 \mathrm{~g} / \mathrm{l} \mathrm{PMP}$ \\
\hline oil+PMP & $100 \%$ Nyflex200 & - & $2 \mathrm{~g} / \mathrm{l} \mathrm{PMP}$ \\
\hline
\end{tabular}

Table 2: Light yield measured using different liquid scintillators. The average calibration constants for PMT 1 and PMT 2 for these data are $13.0 \pm 0.6$ and $12.5 \pm 0.6$ respectively. The errors include both statistical and systematic contributions, added in quadrature.

\begin{tabular}{|l|l|l|l|}
\hline Liquid & PMT 1 [counts] & PMT 2 [counts] & $N_{\text {pe }}$ \\
\hline \hline EJ (old) & $155 \pm 16$ & $147 \pm 15$ & $11.6 \pm 1.2$ \\
\hline EJ (new) & $585 \pm 59$ & $570 \pm 57$ & $46.5 \pm 4.7$ \\
\hline BC-517L & $338 \pm 34$ & $333 \pm 33$ & $28.7 \pm 2.9$ \\
\hline MACRO & $78 \pm 8$ & $79 \pm 8$ & $5.5 \pm 0.5$ \\
\hline oil+PC+PMP & $374 \pm 38$ & $336 \pm 34$ & $27.5 \pm 2.7$ \\
\hline oil+PXE+PMP & $365 \pm 37$ & $391 \pm 39$ & $28.6 \pm 2.9$ \\
\hline LAB+PXE+PMP & $426 \pm 42$ & $439 \pm 44$ & $34.8 \pm 3.4$ \\
\hline LAB+PXE+PPO & $136 \pm 14$ & $132 \pm 13$ & $10.9 \pm 1.1$ \\
\hline LAB+PMP & $405 \pm 41$ & $369 \pm 37$ & $28.1 \pm 2.8$ \\
\hline oil+PMP & $193 \pm 19$ & $161 \pm 16$ & $13.9 \pm 1.4$ \\
\hline
\end{tabular}


Table 3: Light yield measured as a function of the fibre position, the reflecting surface and the fibre diameter. The average calibration constants for PMT 1 and PMT 2 for these data are $14.3 \pm 0.7$ and $12.5 \pm 0.6$ respectively. These measurements were done using the liquid scintillator identified as EJ (old) in the text. The errors include both statistical and systematic contributions, added in quadrature.

\begin{tabular}{|l|l|l|l|l|}
\hline Configuration & Position & PMT 1 [counts] & PMT 2 [counts] & $N_{\text {pe }}$ \\
\hline \hline \multirow{4}{*}{ Al, 1.2 mm } & bot-ctr & $77 \pm 8$ & $79 \pm 8$ & $5.7 \pm 0.6$ \\
\cline { 2 - 5 } & bot-cor & $58 \pm 6$ & $55 \pm 6$ & $3.4 \pm 0.4$ \\
\cline { 2 - 5 } & ctr & $79 \pm 8$ & $80 \pm 8$ & $6.5 \pm 0.8$ \\
\hline \multirow{4}{*}{ Al, 0.5 mm } & bot-ctr & $29 \pm 3$ & $21 \pm 2$ & $2.3 \pm 0.3$ \\
\cline { 2 - 5 } & bot-cor & $28 \pm 3$ & $22 \pm 2$ & $2.1 \pm 0.2$ \\
\cline { 2 - 5 } & ctr & $31 \pm 3$ & $27 \pm 3$ & $1.9 \pm 0.5$ \\
\hline Paint, 1.2 mm & bot-ctr & $193 \pm 19$ & $159 \pm 16$ & $12.8 \pm 1.3$ \\
\cline { 2 - 5 } & bot-cor & $190 \pm 19$ & $141 \pm 14$ & $12.0 \pm 1.2$ \\
\cline { 2 - 5 } & ctr & $155 \pm 16$ & $147 \pm 15$ & $11.6 \pm 1.2$ \\
\hline Paint, 0.5 mm & bot-ctr & $71 \pm 7$ & $60 \pm 6$ & $5.8 \pm 0.7$ \\
\cline { 2 - 5 } & bot-cor & $82 \pm 8$ & $67 \pm 7$ & $5.2 \pm 0.7$ \\
\cline { 2 - 5 } & ctr & $72 \pm 7$ & $68 \pm 7$ & $5.1 \pm 0.5$ \\
\hline
\end{tabular}

Table 4: Light yield measured as a function of the fibre position, for the PVC reflecting surface and the fibre diameter. The average calibration constants for PMT 1 and PMT 2 for these data are $13.2 \pm 0.6$ and $11.7 \pm 0.5$ respectively. These measurements were done using the liquid scintillator identified as EJ (new) in the text. The errors include both statistical and systematic contributions, added in quadrature.

\begin{tabular}{|l|l|l|l|l|}
\hline Configuration & Position & PMT 1 [counts] & PMT 2 [counts] & $N_{\text {pe }}$ \\
\hline \hline PVC, 1.2 mm & bot-ctr & $270 \pm 27$ & $256 \pm 26$ & $20.5 \pm 2.1$ \\
\cline { 2 - 5 } & bot-cor & $270 \pm 27$ & $259 \pm 26$ & $22.1 \pm 2.2$ \\
\cline { 2 - 5 } & ctr & $330 \pm 33$ & $256 \pm 26$ & $25.0 \pm 2.5$ \\
\hline \multirow{3}{*}{ PVC, 0.5 mm } & bot-ctr & $91 \pm 9$ & $81 \pm 8$ & $7.8 \pm 0.8$ \\
\cline { 2 - 5 } & bot-cor & $69 \pm 7$ & $55 \pm 6$ & $4.2 \pm 0.4$ \\
\cline { 2 - 5 } & ctr & $69 \pm 7$ & $65 \pm 7$ & $4.8 \pm 0.5$ \\
\hline
\end{tabular}


Table 5: Ratio of geometric averages and of average number of photo-electrons $N_{\text {pe }}$ for different reflecting surfaces. In all cases, the geometric average and $N_{\text {pe }}$ were averaged over the three fibre positions. These values should be compared to $2.4=1.2 \mathrm{~mm} / 0.5 \mathrm{~mm}$, which is the ratio of fibre diameter.

\begin{tabular}{|l|l|l|}
\hline Reflector & Ratio of geom. av. & Ratio of $N_{\mathrm{pe}}$ \\
\hline \hline $\mathrm{Al}$ & $2.7 \pm 0.2$ & $2.5 \pm 0.2$ \\
\hline Paint & $2.3 \pm 0.2$ & $2.2 \pm 0.2$ \\
\hline Plastic & $3.8 \pm 0.3$ & $4.0 \pm 0.4$ \\
\hline
\end{tabular}



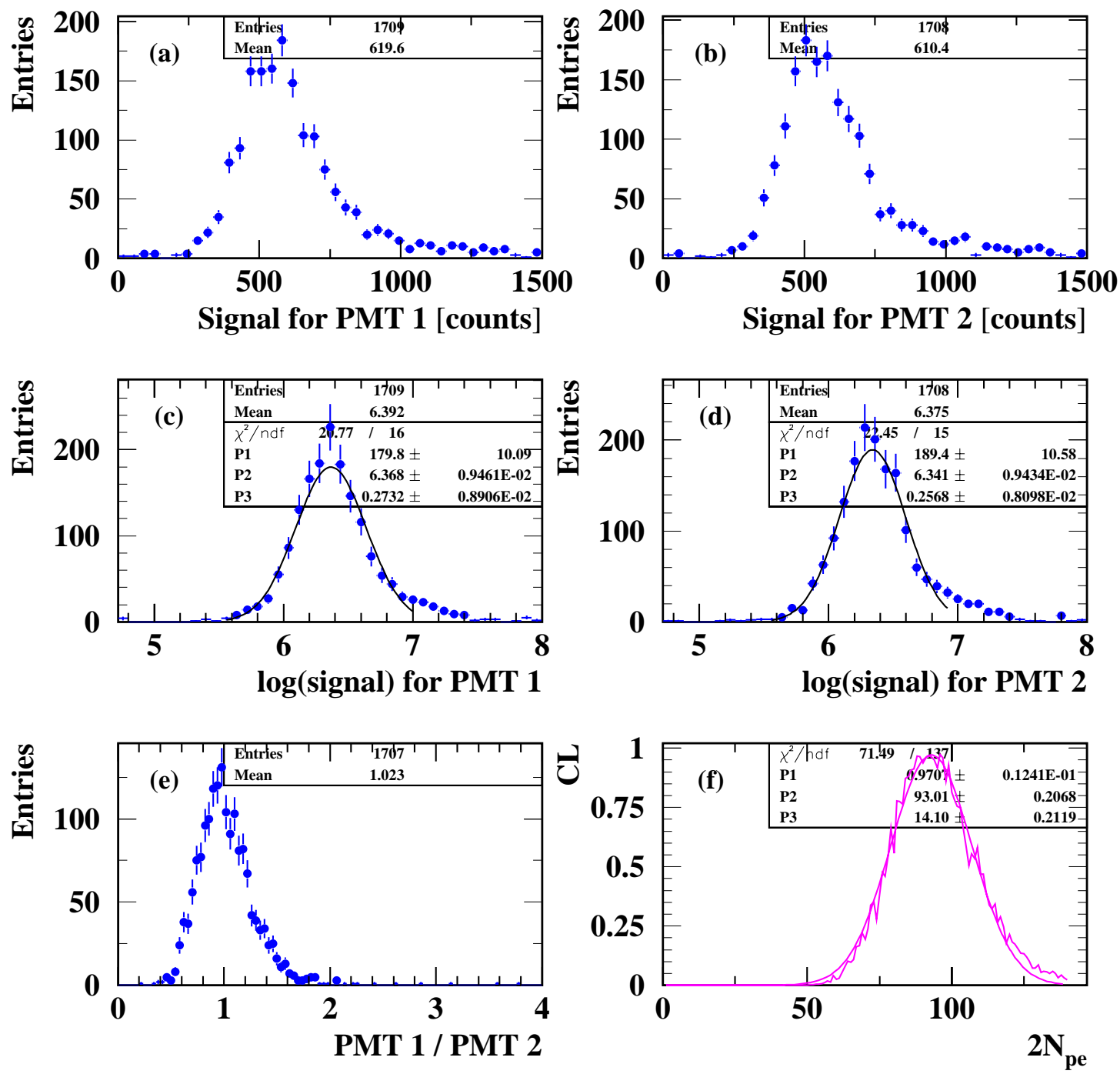

Figure 2: Data taken using the EJ (new) liquid with a $1.2 \mathrm{~mm}$ fibre passing through the centre of the painted tube. Figures (a) and (b) show the pedestal subtracted signals on each PMT. Figures (c) and (d) show the Gaussian fits to $\log$ (signal). Figure (e) shows the distribution of the ratio of the signals. Figure (f) shows the output of the Kolmogorov-Smirnov test as a function of the hypothesis on the average number of photo-electrons. 

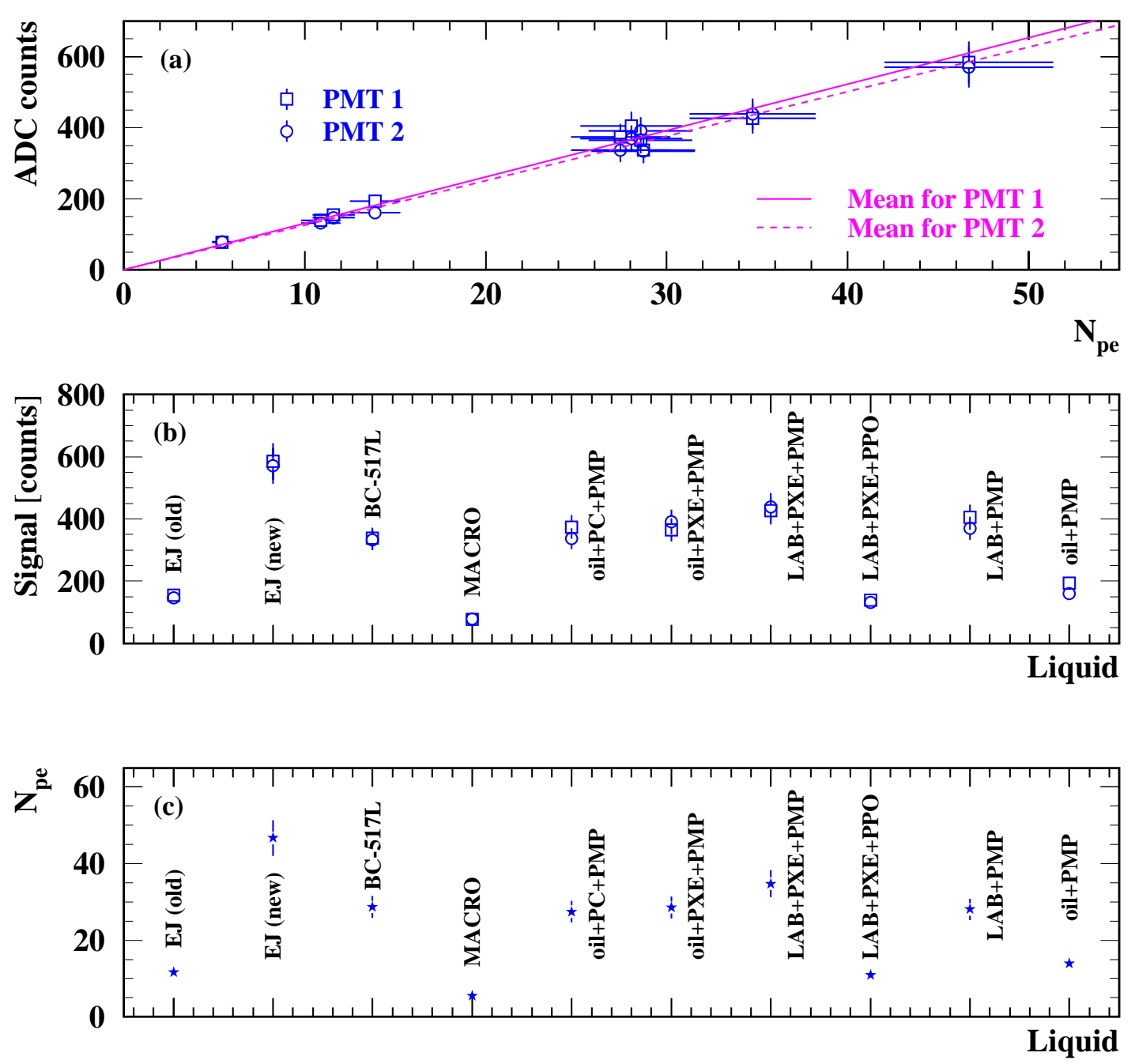

Figure 3: Light yield measured using different liquid scintillators. Figure (a) shows the ADC counts on each PMT as a function of the measured average number of photoelectrons. The average calibration constants for PMT 1 and PMT 2 for these data are $14.0 \pm 0.4$ and $12.0 \pm 0.4$ respectively. Figure (b) shows the signal on each PMT for all liquids, and figure (c) shows the measured average number of photo-electrons on each PMT for each liquid. These measurements were done with a $1.2 \mathrm{~mm}$ fibre running through the centre of the scintillator volume, in the painted tube. The error bars represent both statistical and systematic errors added in quadrature. 

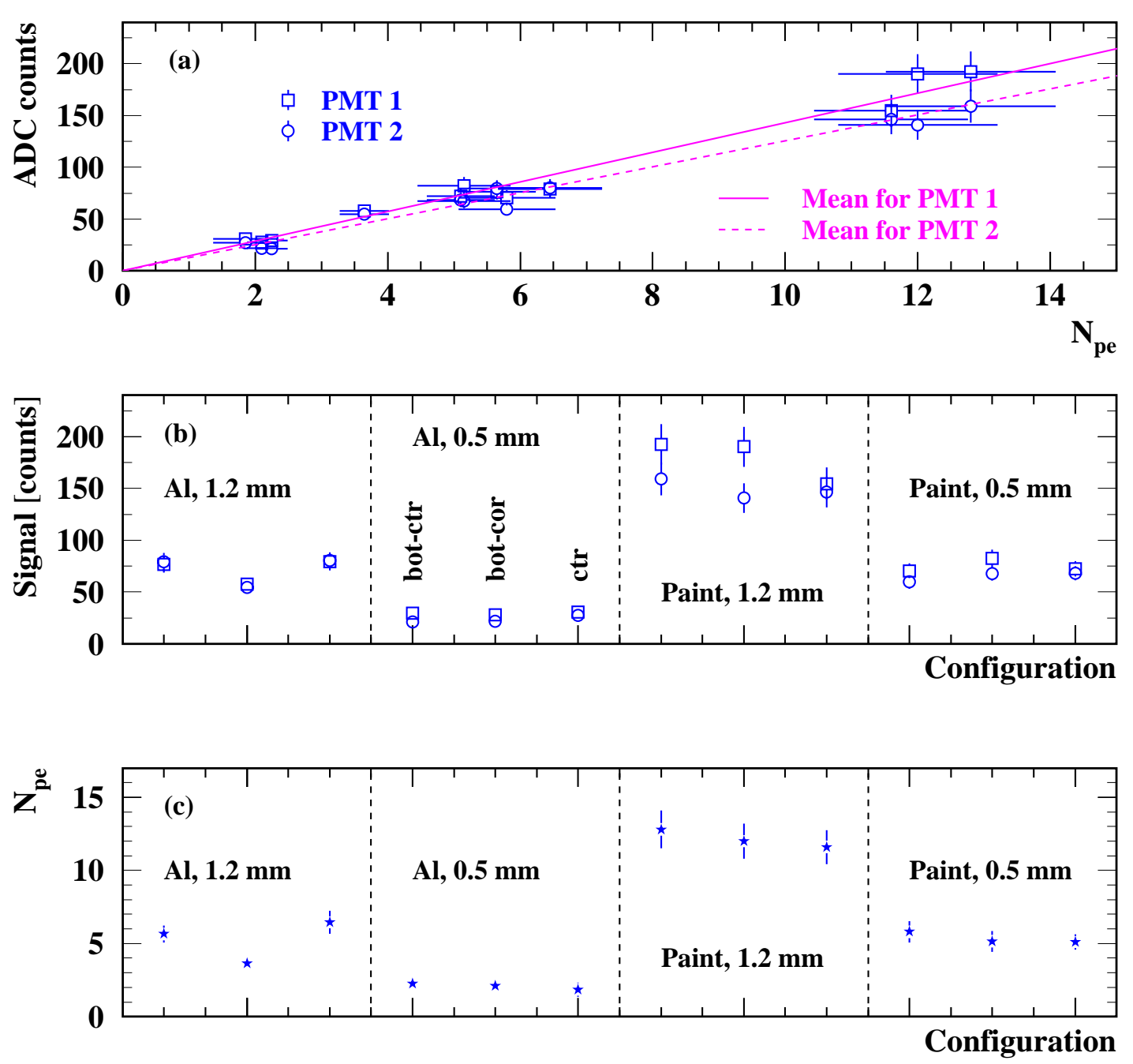

Figure 4: Light yield measured as a function of the fibre position, the reflective surface and the fibre diameter. Figure (a) shows the ADC counts on each PMT as a function of the measured average number of photo-electrons. The average calibration constants for PMT 1 and PMT 2 for these data are $13.1 \pm 0.4$ and $12.5 \pm 0.4$ respectively. Figure (b) shows the signal on each PMT for all configurations, and figure (c) shows the measured average number of photo-electrons on each PMT for each configuration. These measurements were done using the liquid scintillator identified as EJ (old) in the text. The error bars represent both statistical and systematic errors added in quadrature. These samples are separated in groups of three points. The first is for the fibre lying on the bottom surface of the tube, running through the centre. The second is for the fibre running through a corner of the bottom surface. The third is for the fibre running through the centre of the liquid volume. 

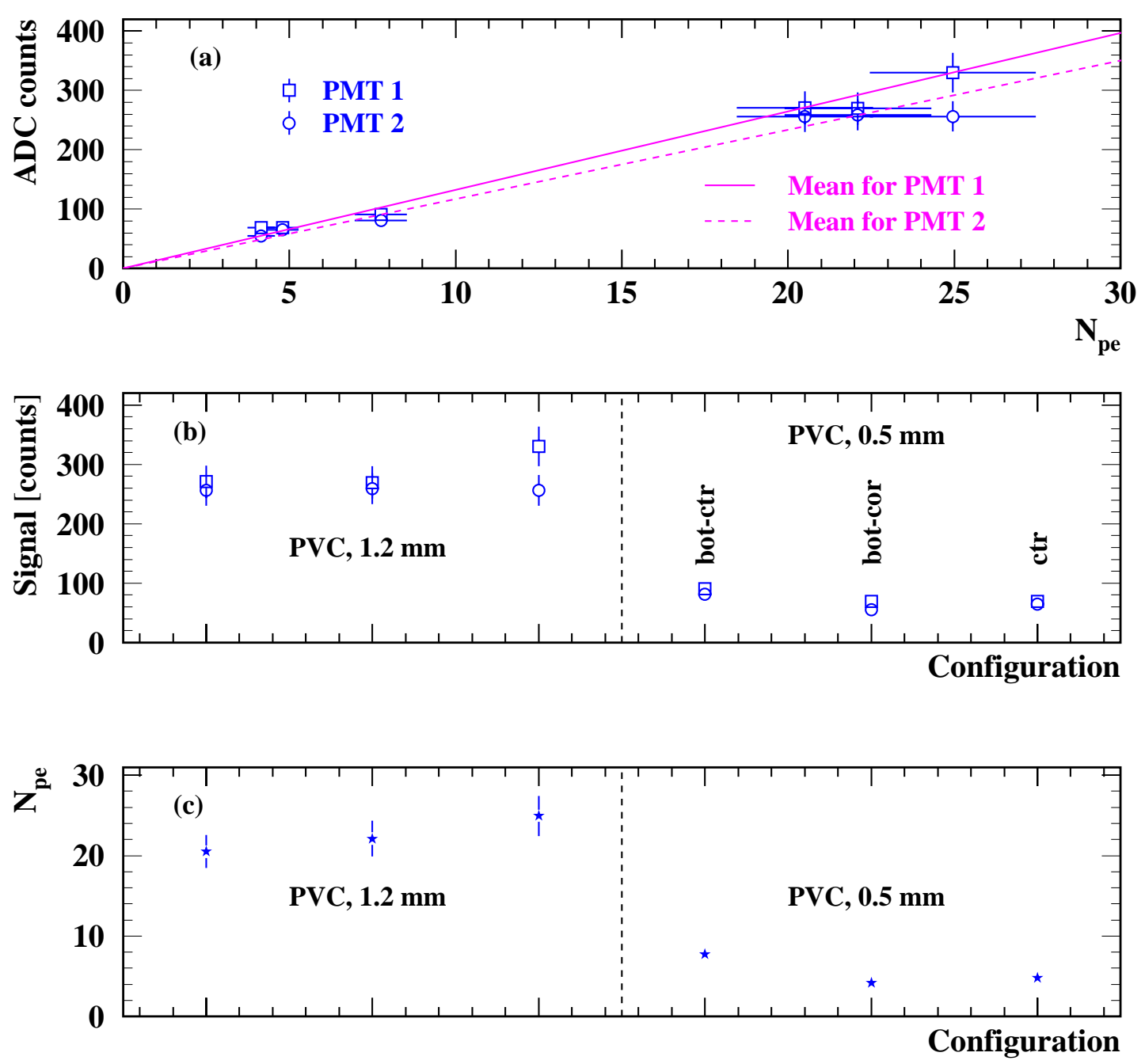

Figure 5: Light yield measured as a function of the fibre position and the fibre diameter for the PVC tube. Figure (a) shows the ADC counts on each PMT as a function of the measured average number of photo-electrons. The average calibration constants for PMT 1 and PMT 2 for these data are $13.2 \pm 0.6$ and $11.7 \pm 0.5$ respectively. Figure (b) shows the signal on each PMT for all configurations, and figure (c) shows the measured average number of photo-electrons on each PMT for each configuration. These measurements were done using the liquid scintillator identified as EJ (new) in the text. The error bars represent both statistical and systematic errors added in quadrature. These samples are separated in groups of three points. The first is for the fibre lying on the bottom surface of the tube, running through the centre. The second is for the fibre running through a corner of the bottom surface. The third is for the fibre running through the centre of the liquid volume. 